\title{
Design of online detection system of gathering machines based on machine vision

\author{
Yingjie TANG
}

\begin{abstract}
Beijing Key Laboratory of Signal and Information Processing for High-end Printing Equipment,
Beijing Institute of Graphic Communication,
\end{abstract}

\#1 Xinghua Avenue, Daxing District, Beijing, China 102600

yingj_tang@263.net

\begin{abstract}
Keywords: Gathering; Machine vision; Detection system.
Abstract: An online detection system is designed for gathering machines to solve the problems arise in collating. The system consists of industrial digital cameras and industrial control computer to form a distributed control system that uses Ethernet to communicate information between host computer and slave computer. Using normalized correlation algorithm for image recognition, IPC can recognize the defective product, and send signals to rejection mechanism to deliver out the unqualified signatures automatically. After repeated tests, the system can promptly identify the defective product such as mixed up signatures, missing signatures and other errors with high recognition rate, and effectively improves the quality of printed products.
\end{abstract}

\section{Introduction}

Post-press processing of magazines and books consists of folding, collating, binding and forming processes. When using gathering machine for collating, errors such as mixed up signatures, upside down signatures, double signatures and missing signatures often occur, these errors are one of the main factors that affect the quality of printed products ${ }^{[1]}$. Now most gathering machine are equipped with detection devices for detecting double signatures and missing signatures, but for common errors as mixed up signatures and upside down signatures, they lack of good detecting methods. For high speed collating, error recognition rate of traditional detection methods such as electronic detection of collating marks, Barcode scanning, electronic determination of the ink density on the print surface and so on is very low ${ }^{[2]}$, that results in relatively high defective rate. So, printing manufacturers urgently need an efficient automatic detection system for signatures inspection to improve product quality.

For this reason, the online detection system based on machine vision for signatures inspection was designed. It can effectively identify the errors as mixed up signatures, upside down signatures, blank signatures and missing signatures, and can eliminate the defective products. The online detection system can improve the quality of printed products and reduce labor costs of the factory $^{[3,4]}$.

\section{System design}

The online detection system for gathering machines based on machine vision uses industrial digital camera to obtain images of signatures; analyzes and processes these images through industrial computer to detect error signatures automatically; and sends detection results to host computer. The host computer will control the rejection mechanism to reject the defective products from the assembly line. The system architecture is shown in Fig.1.

Typically, a gathering machine is equipped with 15 24 collating units, each of which equipped with one industrial digital camera (see Fig.2). The online detection system uses a master-slave distributed control structure. A slave computer controls 4 industrial digital cameras, obtains images of signatures and completes automatically image recognition. Slave computers communicate with host computer through Ethernet to transmit control parameters and image information. 


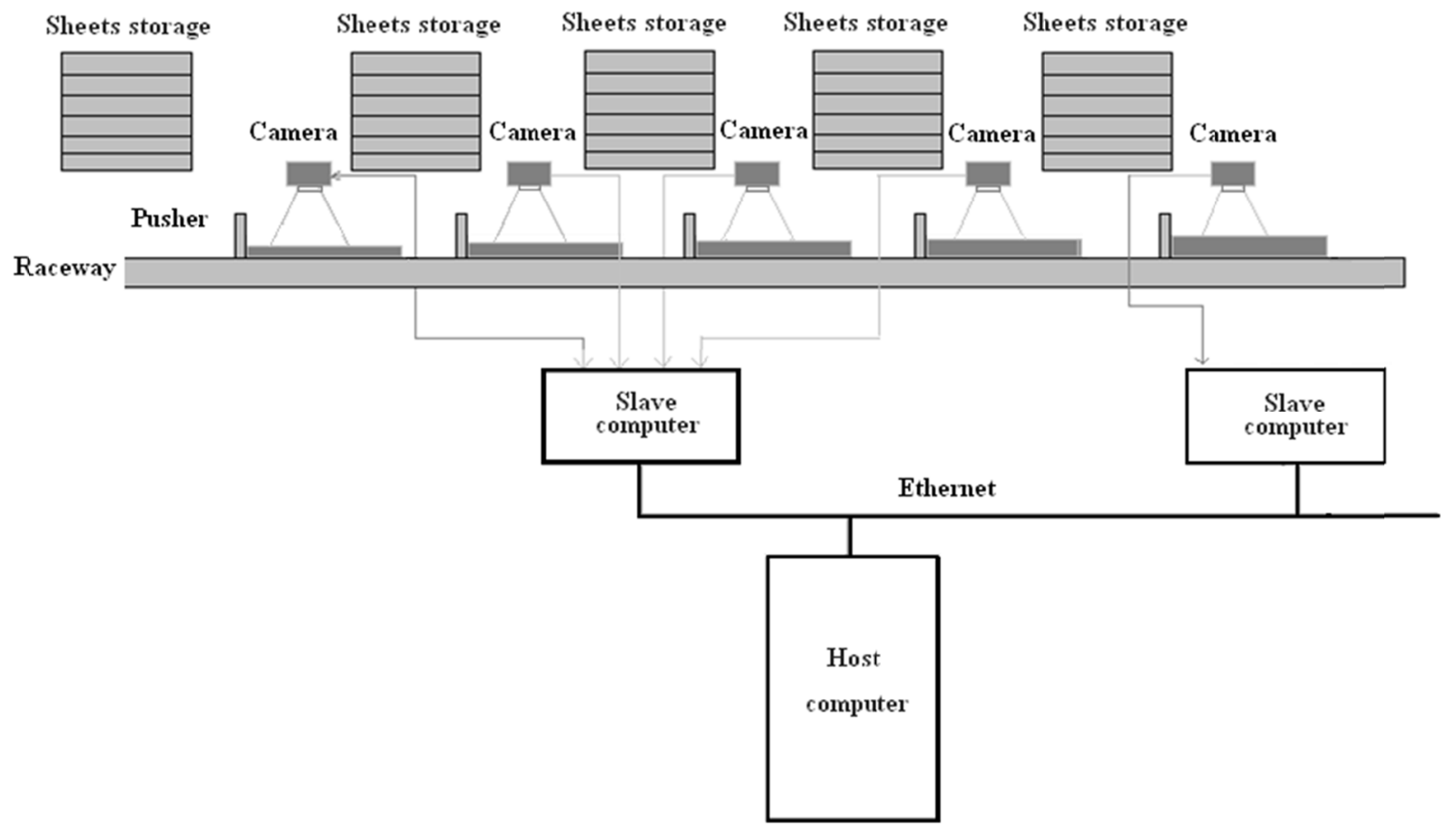

Fig.1 Diagram of detection system

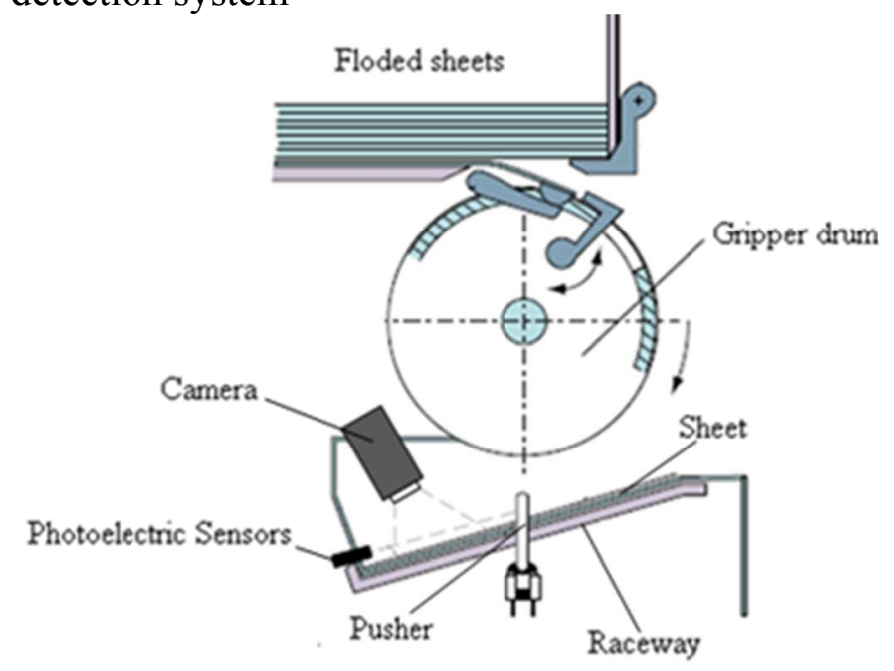

Fig.2 Schematic diagram of detection system for collating unit

Fig.2 is a schematic diagram of detection system. After falling down from sheet storages, signatures are pushed by a pusher to the location just under the industrial digital camera. Once the photoelectric sensor detected the pusher, it sends a signal to trigger the camera shutter, camera captures the image pre-arranged on the signature, slave computer sends the image to host computer, and at the same time performs real-time image recognition to determine whether the signature is qualified or not. If the signature is unqualified, slave computer will send a control signal to the host computer.

Executing elimination algorithm, the host computer controls rejection mechanism to deliver out the unqualified signatures. The host computer can also display in real-time the images of signatures in the collating units, and provide user interface for operators to control the slave computer implementing pre-processing of the images on the signature.

Online detection system work flow:

Start gathering machine, a user interface is displayed on the host computer screen for operators observing the signatures in each collating unit in real-time, selecting the image template by mouse, and sending the image template to slave computers; 
Set the size of image template of each collating unit;

The slave computer processes images captured from 4 industrial digital cameras with corresponding algorithm in time-sharing way. The main process is as follows: image denoising, image displacement, image rotation, template matching and qualified signatures determining. If there is an unqualified signature, the slave computer sends a signal to the host computer;

The host computer calculates the time delay according to the location that errors happen, and sends signals to the rejection mechanism to deliver out the unqualified signatures.

\section{Software design}

The software system of online detection system for gathering machines is divided into host computer software and slave computer software.

Host computer software mainly consists of system parameter setting module, communication module, system status display module and defective product elimination control module. Its main functions are to pre set system parameters, display the working status of each collating unit, communicate with slave computer and control the rejection mechanism. The process of the Host computer is shown in Fig.3.

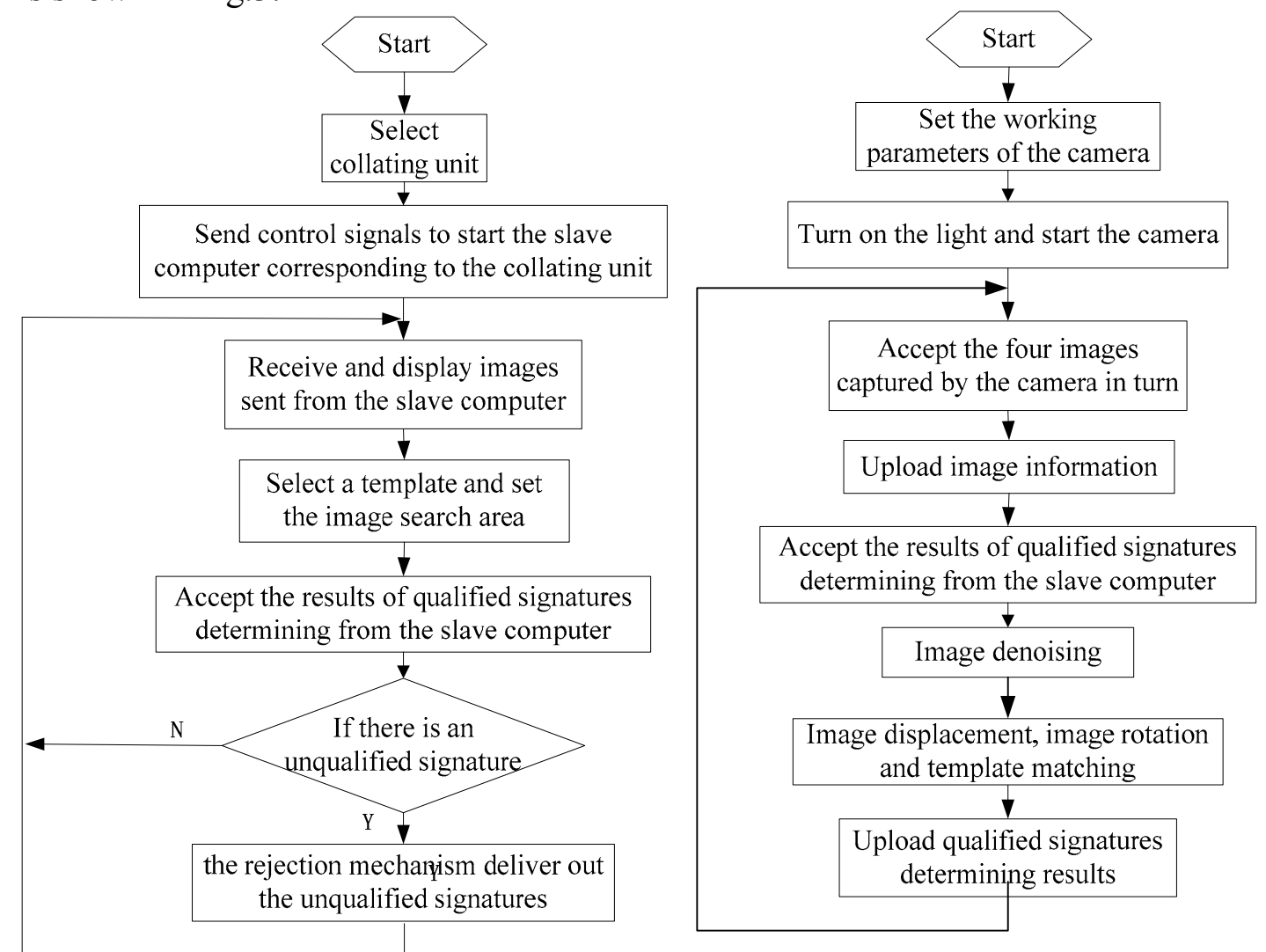

Fig.3 The Host computer flow chart Fig.4 The Slave computer flow chart

Slave computer software mainly consists of communication module, image processing and recognition module. Its main functions are to accept parameters from host computer,turn on the light and start the camera corresponding to the collating unit, obtain images in accordance with the parameters specified, upload images to the host computer, identify signatures of the four collating units in turn, send identify results to the host computer.

The process of the slave computer is shown in Fig.4.

\section{Key algorithms of the detection system}

The key algorithms of the detection system are the image matching algorithm and the defective elimination algorithm. The image matching algorithm uses Normalized Cross-Correlation Matching Algorithm which is a kind of typical algorithm based on gray correlation ${ }^{[5,6]}$. Normalized 
correlation is less sensitive to linear changes in the amplitude of illumination. It has strong robustness and accuracy, and is widely used in image recognition system ${ }^{[7]}$.

$$
\mathrm{NC}(\mathrm{i}, \mathrm{j})=\frac{\sum_{\mathrm{m}=1}^{\mathrm{M}} \sum_{\mathrm{n}=1}^{\mathrm{N}}|\mathrm{S}(\mathrm{m}, \mathrm{n})-\mathrm{E}(\mathrm{S})| \times|\mathrm{T}(\mathrm{m}, \mathrm{n})-\mathrm{E}(\mathrm{T})|}{\sqrt{\sum_{\mathrm{m}=1}^{\mathrm{M}} \sum_{\mathrm{n}=1}^{\mathrm{N}}(\mathrm{S}(\mathrm{m}, \mathrm{n})-\mathrm{E}(\mathrm{S}))^{2} \times \sum_{\mathrm{m}=1}^{\mathrm{M}} \sum_{\mathrm{n}=1}^{\mathrm{N}}(\mathrm{T}(\mathrm{m}, \mathrm{n})-\mathrm{E}(\mathrm{T}))^{2}}}
$$

The similarity formula of normalized correlation gray matching is defined as follows:

Where, $\mathrm{T}$ is a reference template image of size $\mathrm{M} \times \mathrm{N}, \mathrm{G}$ is a real-time acquisition image of size $\mathrm{m} \times \mathrm{n}$, and $\mathrm{m}>\mathrm{M}, \mathrm{n}>\mathrm{N}$. Slide the template $T$ on the image $\mathrm{G}$. the image under the template T refers to subimage $\mathrm{S}, \mathrm{i}$ and $\mathrm{j}$ are coordinates of the pixels in the upper left corner of the subimage of image G.

In $\operatorname{Eq}(1): N C(i, j)$ is the correlation values of $G(i, j) . E(S)$ represents the average of gray value of all pixels in the subimage S; E (T) represents the average of gray value of all pixels in the template $\mathrm{T}$.

Compute the correlation value between the template image $\mathrm{T}$ and the sample image $\mathrm{G}$ in every position. If the correlation value is up to the threshold value, it indicates the matches between the two images ${ }^{[8,9]}$.

Because the sample images may be displaced or tilted at the time of acquisition, so they are processed for many times during image matching. For example, the sample images may be rotated before image matching. Only when the rotation angle of the sample images was-10 degrees $\sim+10$ degrees, and correlation values are not up to the threshold value, the two images can be identified as mismatching.

The design of the defective elimination algorithm is based on the actual structure of the gathering machine. In Fig.5, the gathering machine has 15 collating unit. The photoelectric sensor is placed in close to the location of rejection mechanism. It sends counting signals to the host computer when pushers go through it.

The algorithm is as follows:

CalculateT which is the time needed for a signature moves from the photoelectric sensor position to the deliver out position, and $\mathrm{N}$ which is the numbers of pusher between No.1 collating unit position and the photoelectric sensor position. In Fig.5, $\mathrm{N}$ is 3 .

Establish a $15+\mathrm{N}$ bit data $\mathrm{M}$, and assign an initial value of 0 to $\mathrm{M}$;

IF there is an unqualified signature found in one collating unit, a signal is sent to the host machine through Ethernet by slave computer, then the number of this collating unit is assigned to $\mathrm{S}$, and set the $\mathrm{S}+\mathrm{N}$ th bit of $\mathrm{M}$ to 1 .

At every pulse, all the digits of $\mathrm{M}$ are moved one place to the right(The digital in the last is set to 0 ). When the digital of overflow bit become 1, the timer is started. After delay of $\mathrm{T}$, the host computer sends signal to rejection mechanism, the baffle of the rejection mechanism is put down, the defective product on the gathering chain moves up through the baffle to the area for defective product.

When the host computer withdraws the signal, the baffle lifts back, and qualified signatures enter the next working units along the gathering chain.

Return to step 3, continue. 


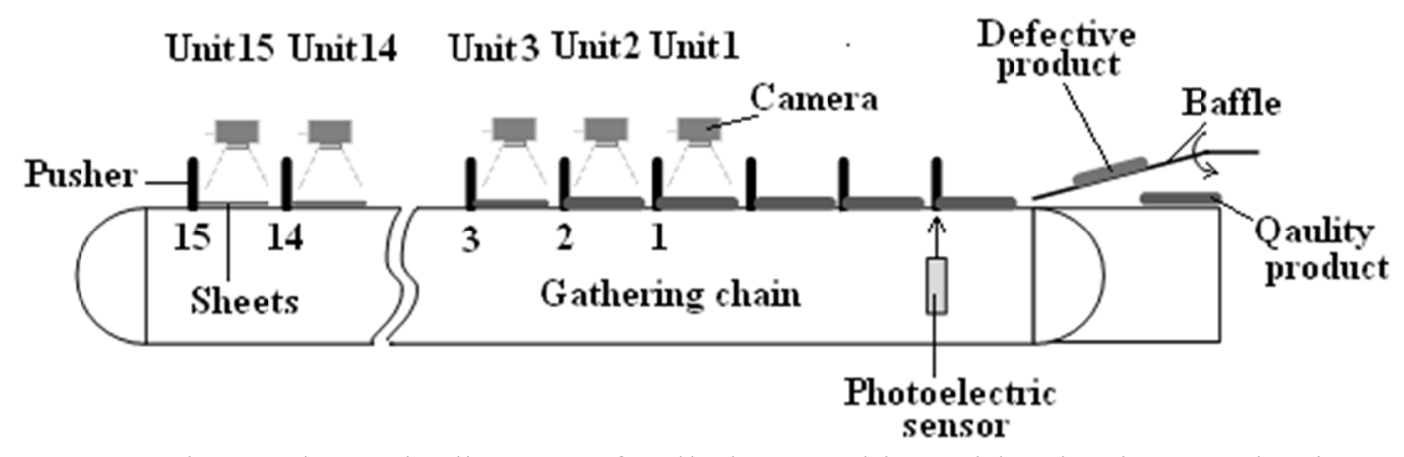

Fig.5 Schematic diagram of collating machine with rejection mechanism

\section{Conclusion}

The online detection system has been tested on PYCD440B collating machine, which produced by Shanghai purple Machinery Co., Ltd.The test results show that the system can effectively identify errors such as mixed up signatures, upside down signatures, double signatures and missing signatures. In the gathering speed of 6000 copies/ hour, effective recognition rate is up to $99 \%$. Implementation of the system can not only improve the quality of the printed products, but also replace manual inspection completely, increase the detection efficiencyand reduce company's labor costs.

\section{References}

[1] RongbaoZhang: Electronic Products. Vol. 10 (2006), p. 89-91.

[2] Helmut Kipphan: Handbook ofPrint Media (Springer Verlag, New York2001).

[3] Wenyu Liand so on:Journalof Xi' an University of Technology, Vol.29 (2013), p.159-163.

[4] Weijun Dai, Yanni Tang: Computer Measurement \& Control, Vol.19(2011), p.2893-2895.

[5] NaYang and so on:Journal of Information Engineering University. Vol.15 (2014), p. 215-164.

[6] Bing Han, Yongming Wang: Acta Armamentarii. Vol. 31 (2010), p. 160-164.

[7] Thevenaz P:IEEE Trans on Image Processing.Vol.9(2000), p. 2083-2099.

[8] Yuan Sunand so on: Journal of Shanghai Jiaotong University. Vol. 34 (2000), p.703.

[9] Songbai Chen: Information and Electronic Engineering.Vol.4 (2006), p. 461-463. 